\title{
Horizontal Transfer of the "Popcorn-Effect" Strain of Wolbachia from Drosophila melanogaster to Stomoxys calcitrans
}

\author{
ENDANG SRIMURNI KUSMINTARSIH
}

\author{
Faculty of Biology, Universitas Jenderal Soedirman, Jalan Dr Soeparno No 63 Grendeng, Purwokerto 53122 \\ Phone: +62-0281-638794, Fax: 62-0281-631700,_E-mail: endang_sk@lycos.com
}

\begin{abstract}
The Wolbachia containing haemolymph from Wolbachia infected Drosophila third/fourth instar larvae was transferred through microinjection into 5-days-old pupae of the blood sucking stable fly, Stomoxys calcitrans. There was no previous record of Wolbachia being present in S. calcitrans. From a total of 682 emerging adults, 236 were females and of these, seven females were tested positive (approximately 3\%) for Wolbachia infection. Using electron microscopy, it was shown that Wolbachia were present in the muscle tissues of $S$. calcitrans.
\end{abstract}

Key words: Drosophila melanogaster, Stomoxys calcitrans, 'popcorn-effect', haemolymph, microinjection

Wolbachia infection was first discovered in mosquitoes (Hertig 1936). Since then, these intracellular bacteria have been found in a variety of insect species, mites and isopods (Rousset et al. 1992; Breeuwer and Jacobs 1996; Werren 1997; Grenier et al. 1998). Wolbachia can induce different effects in the reproductive organs of its hosts. These include cytoplasmic incompatibility (CI) in isopods, insects and mites (Breeuwer and Jacobs 1996), parthenogenesis/thelytoky (T) in parasitoid insects (Stouthamer et al. 1990), feminisation (F) in isopods (Juchault et al. 1994), male-linked lethality in Drosophila bifasciata and the butterfly Acraea encedon (Hurst et al. 1999 and 2000), and the 'popcorn-effect' in D. melanogaster in which an early death of the insect occurs (Min and Benzer 1997). A sudden massive degeneration of the fly's cells occurs in response to bacterial multiplication. The bacteria are present in low numbers during development through the embryonic, larval and pupal stages. As soon as the adult flies emerge, the bacteria start to multiply rapidly, causing sudden death of their host. It might be possible to make use of the 'popcorn-effect' induced by Wolbachia to control insect vectors of important human diseases in which the pathogen or parasite is transmitted at the end of the insect's life span after it has taken three to four blood meals. In the future, this may play a vital role in the prevention of arthropod-borne diseases; disease transmission depends on the older insect and Wolbachia infection has been found to shorten the life span of insects.

Wolbachia is maternally inherited. However, there is strong evidence for horizontal transfer between species that are distantly related, based on non-congruence of host and bacterial phylogenies (O’Neill et al. 1992; Rousset et al. 1992; Werren et al. 1995). Comparative molecular phylogenies of 20 parthenogenetic Trichogramma populations and their symbiotic Wolbachia suggest the occurrence of occasional horizontal transmission (Schilthuizen and Stouthamer 1997). This is a good indication that individual Wolbachia species or strains are not restricted to a single host. The 'popcorneffect'-inducing Wolbachia causes a reduction in insect life span, so if this strain could be transferred to insects that are disease vectors, it might be an effective vector control. For example, adult Anopheles gambiae, which transmits Plasmodium spp. (causing malaria), has a life expectancy of
15 days (Garrett-Jones and Shidrawi 1969) whilst Plasmodium has an incubation period of 11 days (Baker 1966). Similarly, adult Aedes trivittatus has a life expectancy of about 25 days in the laboratorium (Christensen 1978) whilst the parasite it transmits (Dirofilaria immitis) has an incubation period of 16 days at $22.5^{\circ} \mathrm{C}$ (Christensen and Hollander 1978). They will only be infectious and pathogens transmitted after the host has taken at least three to four blood meals. If the insect was infected with the 'popcorn-effect'-inducing Wolbachia, the insect will experience early and sudden death in its adult stage perhaps preventing disease transmission to humans.

Experimental transfers have been performed, in which Wolbachia has been transferred by microinjection (within and between species, between families, and between orders (Boyle et al. 1993; Braig et al. 1994; Chang and Wade 1994; Juchault et al. 1994; Karr 1994; Rousset and Stordeur 1994; Rigaud and Juchault 1995; Sinkins et al. 1995; Clancy and Hoffmann 1997; Dunn and Rigaud 1998; Grenier et al. 1998; Poinsot et al. 1998; Van Meer and Stouthamer 1999; Fujii et al. 2001). The interfamily transfer of Wolbachia was demonstrated by Braig et al. (1994). They found that bacteria from the mosquito Aedes albopictus could be transferred by microinjection into uninfected embryos of $D$. simulans, conferring complete CI on the adults. Recently, embryonic microinjection protocol for Wolbachia transfection have been developed for the mosquitoes A. albopictus (Xi et al. 2006) and A. aegypti (Xi et al. 2005), as well as Wolbachia transfer protocols based on injection of symbionts directly into adult Drosophila and Aedes mosquitoes (Frydman et al. 2006; Ruang-Areerate and Kittayapong 2006). Grenier et al. (1998) successfully transferred the symbiote within the Trichogrammatidae. However, there is no study to date on horizontal transfer of the 'popcorn-effect'. To investigate whether the 'popcorn-effect' phenotype is specific to Drosophila or whether it can be transmitted to another species, the popcorn-effect inducing Wolbachia was transferred from $D$. melanogaster by microinjection into uninfected $S$. calcitrans pupae. There is no previous record of Wolbachia being present in S. calcitrans.

To verify the presence of Wolbachia in newly infected individuals of $S$. calcitrans, the tissues from $S$. calcitrans which tested positive for Wolbachia by PCR were then 
examined by electron microscopy. This is to confirm that the positive PCR amplification represented the identification of real Wolbachia, and not a fortuitous PCR amplification of genomic DNA.

\section{MATERIALSAND METHODS}

Microinjections. Stomoxys calcitrans was obtained from Prof Lehane's and D. melanogaster larvae harbouring Wolbachia 'popcorn-effect' was obtained from Dr. Braig's laboratory School of Biological sciences, University of Wales, Bangor, United Kingdom. Microinjection experiments using cell micro-injector PM 1000, a D. melanogaster fourth instar larva, derived from a previously established colony, was placed in front of the microcapillary needle and punctured. Subsequently, the haemolymph was drawn into the needle via capillary action of the filament. A 5-day-old S. calcitrans pupa was placed in front of the needle and punctured with the use of the macro- and microcontrols. The D. melanogaster haemolymph in the needle flowed into the pupa when the control pedal was pressed. The microcapillary needle was then removed. If the needle broke or if backflow into the needle occurred, then the pupa was discarded. A new needle was used for each transfer. In order to ensure that all pupae used in this experiment were of the same age (5-day-old pupae), all stock used was 17 days old from egg collection. The pupae were kept on moist cotton wool (checked daily, water being added if necessary).

Rearing, Collection, and Killing of Flies. After microinjection the pupae were put in a plastic cup $(10.5 \mathrm{~cm}$ diameter, $4 \mathrm{~cm}$ height) containing moist cotton wool. Humidity was maintained by regular addition of water. The plastic cups were housed in net cages $(26 \mathrm{~cm}$ length, $26 \mathrm{~cm}$ width and $26 \mathrm{~cm}$ height) at $25^{\circ} \mathrm{C}$. The number of pupae in every plastic cup container depended on the number of pupae that were injected in the same day $(187,69,173,48,30$, 184, 199, 119, 87, 169, 109, 109, 109, 116 and 37). The cages were placed on paper in order to facilitate egg collection (next generation). Adults emerged after several days and were allowed to mate. Adult flies were fed daily with pig's blood (by placing cotton wool, which was soaked in the pig's blood, on top of the net cage). Eggs were transferred from the paper into a plastic cup container $(10 \mathrm{~cm}$ diameter and $4 \mathrm{~cm}$ height) containing larval feeding medium after 28 days from emergence, adult flies were killed and the females tested for Wolbachia infection. The period of 28 days after emergence, is the longest possible time before risking loosing any flies due to natural mortality. The flies were not killed sooner in order to allow the bacteria to grow in the new host. The period of 28 days gave the female flies the possibility to lay eggs so that the line would continue to the next generation. As Wolbachia are maternally inherited, the bacteria would be transmitted to the progeny just through the females, not the males.

DNA Extraction, Electrophoresis, and Electron Microscopy. The procedure of DNA extraction was using the DNeasy tissue kit (Qiagen). The extracted DNA from the adult Stomoxys calcitrans female was analysed using the polymerase chain reaction (PCR) to determine evidence of Wolbachia infection. The procedure was according to the manufacture instructions (Promega). One master mix was used to detect Wolbachia (using Wolbachia surface protein wsp gene). Forward (w81F: 5'-TGG TCC AAT AAG TGA TGA AGAAAC-3') and the reverse primer (w691R: $5^{\prime}$-AAAAAT TAA ACG CTA CTC CA-3) were designed to amplify a fragment of $610 \mathrm{bp}$ (Braig et al. 1998); as a control to detect mitochondria, forward primer $12 \mathrm{SbiF}\left(5^{\prime}\right.$-AAGAGC GAC GGG CGA TGT GT-3') and reverse primer $12 \mathrm{SaiR}$ (5'-AAA CTA GGA TTA GAT ACC CTA TTA T-3') were used. The microcentrifuge tubes were then placed in a thermal cycler (Technogene). An initial denaturation at $95^{\circ} \mathrm{C}$ for 5 minutes was followed by 35 cycles of $95^{\circ} \mathrm{C}$ for 1 minute, $55^{\circ} \mathrm{C}$ for 1 minute and $72^{\circ} \mathrm{C}$ for 2 minutes: followed by $72^{\circ} \mathrm{C}$ for 10 minutes and finally $4^{\circ} \mathrm{C}$.

To verify the presence of Wolbachia in individuals of $S$. calcitrans, which were infected by microinjection of haemolymph from $D$. melanogaster infected with the popcorn strain of Wolbachia, the tissues from S. calcitrans tested positive for Wolbachia by PCR were further examined by electron microscopy. This is to confirm that the positive PCR amplification represented the identification of real Wolbachia, not fortuitous PCR amplification of genomic DNA. The tissues (muscle, testes, ovaries, brain and eyes) were taken from flies dissected in PBS (phosphate buffer saline) and put in Karnofsky's fixative (Glauert and Lewis 1998) consisting of $0.4 \%$ paraformaldehyde and $0.16 \%$ glutaraldehyde in sodium cacodylate, $10 \% \mathrm{w} / \mathrm{v}$ sucrose, $\mathrm{pH}$ 7.2 for $1 \mathrm{~h}$, then washed twice using PBS. The tissues are then put in $1 \%$ osmium tetroxide plus cacodylate buffer (1:1) for one hour, washed twice with water and put in $2 \%$ uranyl acetate and kept in the fridge $\left(4^{\circ} \mathrm{C}\right)$ overnight. After fixation the material was dehydrated using serial ethanol changes, and embedded in the resin. A glass knife was used to shape and cut the embedded sample in the piramitome (LKB Leica) and ultratome (LKB Leica). The sections were collected on grids (pioloform coated nickel 200 mesh grids) and then stained with lead citrate for 6 minutes. Sections were observed using a Philips EM 60 kV.

\section{RESULTS}

From a total of 682 emerging adults, 236 were females and of these, seven females (approximately 3\%) tested positive for Wolbachia infection. Fig 1 and 2 show gel electrophoreses of injected female $S$. calcitrans testing positive for Wolbachia infection. The first lane was a one kilobase ladder, second lane was Wolbachia positive control from D. melanogaster infected with 'popcorn-effect'. Therefore, the next lanes, which had the same size of band as the $D$. melanogaster positive control, indicated that $S$. calcitrans were Wolbachia positive lane 4, 6, 8, 10, 18 and 20 in Fig 1 and lane 4 in Fig 2. Mitochondrial primers (12SbiF and 12SaiR) were used for checking whether the conditions for the PCR were appropriate in every sample. Using electron microscopy it was shown that Wolbachia were present in the muscle tissues of $S$. calcitrans (Fig 3 and 4).

To provide evidence that $S$. calcitrans did not contain bacteria before injection with Wolbachia, the muscle tissue was examined by PCR and electron microscope. Fig 5 shows gel electrophoresis of $S$. calcitrans testing negative for 


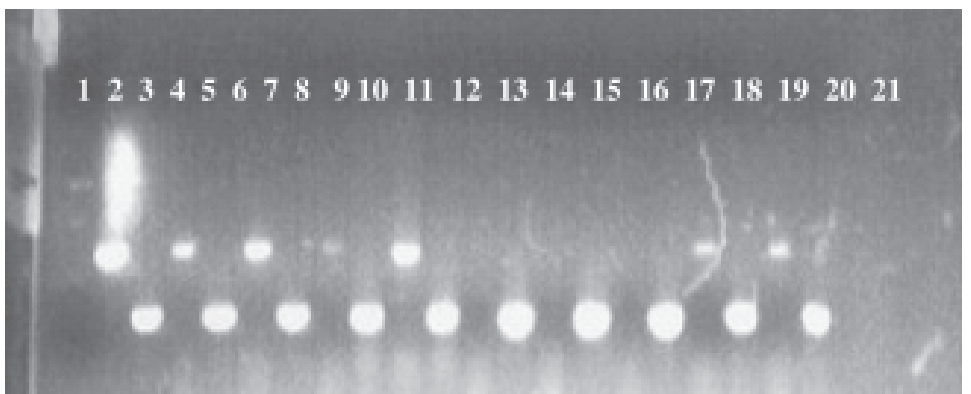

Fig $1 \mathrm{Gel}$ electrophoresis of Stomoxys calcitrans testing positive for Wolbachia infection. Lane 1, $1 \mathrm{~kb}$ ladder; lane 2, positive control for Wolbachia from Drosophila melanogaster; lane 3, positive control for mitochondria from D. melanogaster; lane 4, 6, 8, 10, 18 and 20. Wolbachia positive results from S. calcitrans; lane 5, 7, 9, 11, 13, 15, 17, 19 and 21, Mitochondria from S. calcitrans; lane $12,14,16$. Wolbachia negative results from $S$. calcitrans.

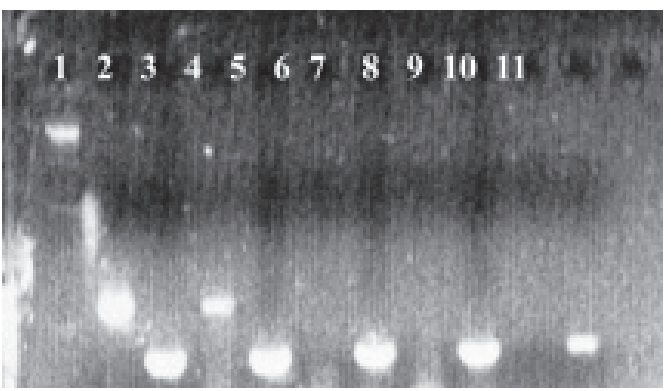

Fig 2 Gel electrophoresis of Stomoxys calcitans testing positive for Wolbachia infection. Lane 1, $1 \mathrm{~kb}$ ladder; lane 2, positive control for Wolbachia from D. melanogaster; lane 3, positive control for mitochondria from D. melanogaster; lane 4, Wolbachia positive result from S. calcitrans; lane 5, 7, 9, and 11. Mitochondria from $S$. calcitrans; lane 6, 8, and 10. Wolbachia negative from S. calcitrans.

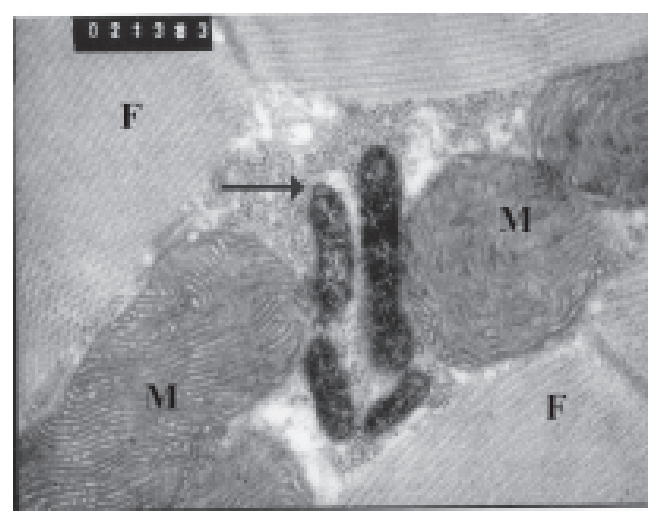

Fig 3 Stomoxys calcitrans muscle tissue $(51,000 \mathrm{X})$. Using electron microscopy it was shown that Wolbachia were present in the muscle tissues of $S$. calcitrans. M, mitochondria and F, fibril of muscle. Arrow: Wolbachia.

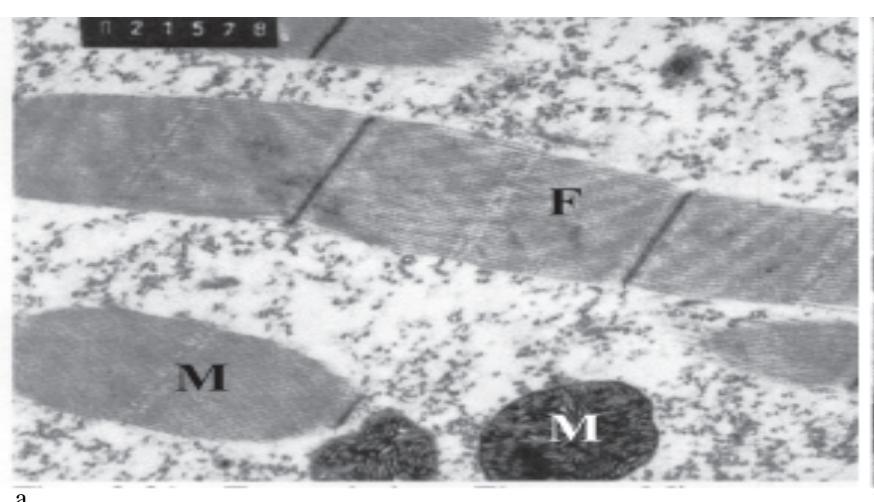

$\mathrm{a}$

Figs 6 Muscle of uninfected Stomoxys calcitrans before injection (20000 X): a, a horizontal section and b, a transversal section. M, mitochondria and F, fibril of muscle. There is no Wolbachia in the tissue.

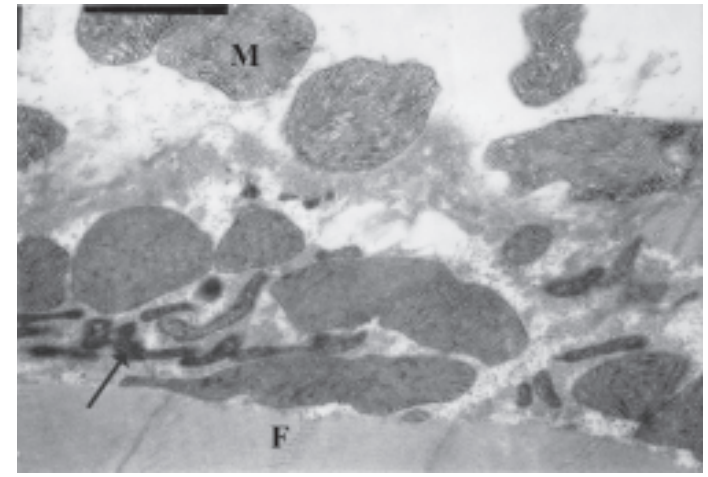

Fig 4 Stomoxys calcitrans muscle tissue $(19,000 \mathrm{X})$. M, mitochondria and F, fibril of muscle. Arrow: Wolbachia.

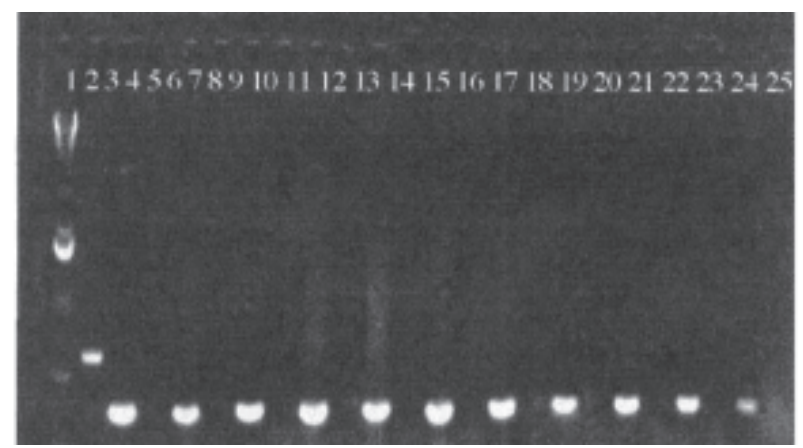

Fig 5 Gel electrophoresis of Stomoxys calcitrans testing negative for Wolbachia infection before injecting. Lane 1, $1 \mathrm{~Kb}$ ladder; lane 2. Positive control for Wolbachia; lane 3, 5, 7, 9, 11, 13, 15, 17, 19, 21, 23, 25 mitochondria primer of $S$. calcitrans; lane 4, 6, 8, 10, 12, $14,16,18,20,22,24$ Wolbachia primer of $S$. calcitrans there were no bands indicating negative of Wolbachia.

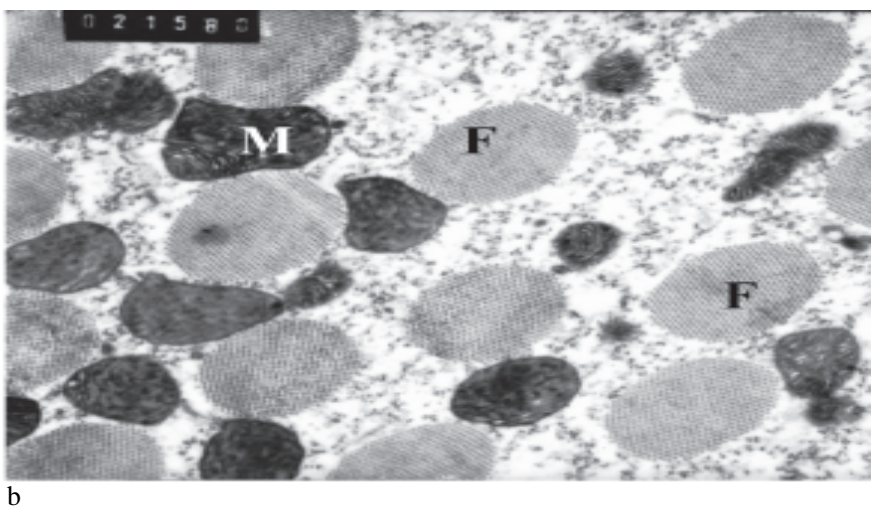


Wolbachia infection before injecting and Fig 6 show muscle tissues of S. calcitrans, where no Wolbachia or any other bacterial structures were detected. This indicated that uninjected (normal slide) S. calcitrans were negative for Wolbachia.

\section{DISCUSSION}

From 236 females, only seven females (approximately 3\%) tested positive for Wolbachia infection. There are several possible reasons for why such a low number of positive results were obtained. Firstly, the Wolbachia density might have been too low to produce an infectious dose in the pupae, resulting in elimination of the inoculum. Secondly, that the interaction between Wolbachia symbiont from $D$. melanogaster and the new host genome of $S$. calcitrans is unfavourable (Kondo et al. 2005). Therefore, the success of transmission for the bacteria are influenced by several factors, including bacterial density and host genotype (Breeuwer and Werren 1993; Bourtzis et al. 1996; Grenier et al. 1998; Kondo et al. 2005; Duron, et al. 2006; Mouton et al. 2006). Thirdly, the pupae might have been too young. Young pupae have a very up-regulated immune system whereas older pupae (which are protected by an additional exoskeleton) exhibit a highly down-regulated immune system (Braig HR 2009, personal communication). If the pupae are too young at the time of infection, the inoculum might be destroyed by the immune system regardless of its dose. However, if the pupae are much too old, the barrier between haemolymph and germ line tissue might become unpenetrable for Wolbachia. Strong somatic infections might result but permanent, inheritable infections might not be established. Even a germ line infection requires a certain threshold density of bacteria before a productive infection in the next generation can be secured. Reaching this threshold for an endosymbiont that has been adapted to a different host for thousands and thousands of generations might constitute an immense barrier to be overcome.

Although PCR is far from quantitative, some of the Wolbachia signals from females in lanes 4, 6, 8, 10, 18 and 20 in Fig 1 and lane 4 in Fig 2 were similar the intensity of the Wolbachia signal of the infected donor, being the 'popcorneffect' of D. melanogaster. The strong signal of females (aged 28 days) suggests a productive infection. The PCR assay is not sensitive enough to pick up the inoculum. Lane 4 is presumably an example of a weak infection. Unfortunately, even the six strongest infections did not lead to any inheritance. Similar results have been obtained in flour beetles (Tribolium confusum) in which the infection has been lost in the second generation as well (Chang and Wade 1994). van Meer and Stouthamer (1999) transferred Wolbachia from Muscidifurax uniraptor (Hymenoptera), which harbours parthenogenesis-inducing Wolbachia to D. simulans (Diptera) without establishing a stable infection. For $S$. calcitrans that were positive for Wolbachia after transferring these bacteria by microinjection, no specific effects on the host were detected and the bacteria were not maintained stably. However, investigation by electron microscopy showed that the bacteria were present in the tissues of infected $S$. calcitrans such as the thoracic muscle.
This indicates that Wolbachia develops in the tissues of the new host. It was assumed that the bacteria did not reach the reproductive organs when injected, thereby causing no maternal transmission to the next generation.

Furthermore, host-symbiont interactions are important for success in the establishment of an infection. Another example of a failed transfer of Wolbachia has been obtained for the isopods Chaetophiloscia elongata and Armadillidium vulgare, which are distantly related species. However, Wolbachia could be transferred between closely related species of isopods (A. nasatum to A. vulgare) (Juchault et al. 1994). On the other hand, phylogenetic work on Wolbachia (Werren et al. 1995) provides evidence that, at least in some cases, this bacterium has successfully transferred over large phylogenetic distances in its interspecific movement. It remains unknown what further permits particular inter-family transfers to occur successfully, other than time.

Using electron microscopy it was shown that Wolbachia were present in the muscle tissues of S. calcitrans. The most common form observed was an elongated cylinder with convex ends typical of bacterial rods; it is the most common shape of bacteria, after all, the word bacterium means rod or staff. The second form of Wolbachia that had been encountered was a barrel shape, oval like an egg and showed a maximum diameter along its length. The presence of Wolbachia in S. calcitrans was reported for the first time in this study. It was confirmed by EM following a positive PCR result. However, although there were positive results in S. calcitrans, no permanent establishment of Wolbachia from D. melanogaster occurred in S. calcitrans and the Wolbachia could not be detected in the next generation. A reason for the infection not being stable is that Wolbachia may not be physiologically adapted to its new host. The Wolbachia found in somatic muscle tissues of S. calcitrans, were morphologically similar to the Wolbachia present in D. melanogaster

\section{ACKNOWLEDGEMENT}

This work was supported by DUE Batch II, Universitas Jenderal Soedirman, Purwokerto, Indonesia. I would like thank to MJ Lehane and Henk R Braig, School of Biological Sciences, University of North Wales, Bangor, United Kingdom for guidance and providing facilities, and thank to Alison Bell for the help with electron microscopy.

\section{REFERENCES}

Baker JR. 1966. The fine structure of the exoerythrocytic stages of plasmodium fallax. Trans R Soc Trop Med Hyg 28:355-73.

Bourtzis K, Nirgianaki A, Markakis G, Savakis C. 1996. Wolbachia infection and cytoplasmic incompatibility in Drosophila species. Genetics 144:1063-73.

Boyle L, O'Neill SL, Robertson HM, Karr TM. 1993. Interspecific and intraspecific horizontal transfer of Wolbachia in Drosophila. Science 260:1796-9.

Braig HR, Guzman H, Tesh RB, O'Neill SL. 1994. Replacement of the natural Wolbachia symbiont of Drosophila simulans with a mosquito counterpart. Nature 367:453-5. 
Braig HR, Zhou W, Dobson SL, O’Neill SL. 1998. Cloning and characterization of a gene encoding the major surface protein of the bacterial endosymbiont Wolbachia pipientis. J Bacteriol 180:2373-8

Breeuwer JAJ, Jacobs G. 1996. Wolbachia: intracellular manipulators of mite reproduction. Exp Appl Acarol 20:421-34.

Breeuwer JAJ, Werren JH. 1993. Cytoplasmic incompatibility and bacterial density in Nasonia vitripennis. Genetics 135:565-74.

Chang NW, Wade MJ. 1994. The transfer of Wolbachia pipientis and reproductive incompatibility between infected and uninfected strains of the flour beetle Tribolium confusum by microinjection. Microbiology 40:978-81.

Christensen BM. 1978. Dirofilaria immitis: Effect on the longevity of Aedes trivittatus. Exp Parasitol 44:116-23.

Christensen BM, Hollander AL. 1978. Effect of temperature on vector-parasite relationships of Aedes trivittatus and Dirofilaria immitis. Proc Helminthol Soc Washington 45:115-9.

Clancy DJ, Hoffmann AA. 1997. Behavior of Wolbachia endosymbionts from Drosophila simulans in D. serrata, a novel host. Am Natural 149:975-88.

Dunn AM, Rigaud T. 1998. Horizontal transfer of parasitic sex ratio distorters between crustacean hosts. Parasitology 117:15-9.

Duron OP, Fort M, Weill. 2006. Hypervariable prophage WO sequences describe an unexpected high number of Wolbachia variants in the mosquito Culex pipiens. Proc R Soc London B 273:495-502.

Frydman HM, Li JM, Robson DN, Wieschaus E. 2006. Somatic stem cell niche tropism in Wolbachia. Nature 441:509-12.

Fujii Y, Kageyama D, Hoshizaki S, Ishikawa H, Sasaki T. 2001. Transfection of Wolbachia in Lepidoptera: the feminizer of the adzuki bean borer Ostrinia scapulalis causes male killing in the Mediterranean flour moth Ephestia kuehniella. Proc R Soc London B 268:855-9.

Garrett-Jones C, Shidrawi GR. 1969. Malaria vectorial capacity of a population of Anopheles gambiae: an exercise in epidemiological entomology. Bull WHO 40:531-45.

Glauert AM, Lewis P. 1998. Biological Specimen Preparation for Transmission Electron Microscopy. London: Portland Pr.

Grenier S, Pintureau B, Heddi A, Lassabliere F, Jager C, Louis C, Khatchadourian. 1998. Successful horizontal transfer of Wolbachia symbionts between Trichogramma wasps. Proc R Soc London B 265:1441-5.

Hertig M. 1936. The Rickettsia, Wolbachia pipientis and associated inclusions of the mosquito Culex pipiens. Parasitology 28:45390.

Hurst GDD, Jiggins FM, Schulenberg HG von der, Bertrand D, West ST, Goriacheva II, Zakharov IA, Werren JH, Stouthamer R, Majerus MEN. 1999. Male killing Wolbachia in two species of insect. Proc R Soc London B 266:735-40.

Hurst GDD, Johnson AP, Schulenburg JHG von der, Fuyama Y. 2000. Male killing Wolbachia in Drosophila: A temperature sensitive trait with a threshold bacterial density. Genetics 156:699-709.

Juchault P, Frelon M, Bouchon D, Rigaud T. 1994. New evidence for feminizing bacteria in terrestrial isopods: evolutionary implications. Comptes Rendus Acad Sci Paris III 317:225-30.

Karr TL. 1994. Giant steps sideways-the horizontal transfer of a bacterial endosymbiont that is intimately associated with reproductive isolation in insects is now feasible and may, in principle, lead to new strategies for biological control. Curr Biol 4: 537-40.

Kondo NM, Shimada, Fukatsu T. 2005. Infection density of Wolbachia endosymbiont affected by co-infection and host genotype. Biol. Lett 1:488-91.

Meer MMM van, Stouthamer R. 1999. Cross-order transfer of Wolbachia from Mucidifurax uniraptor (Hymenoptera: Pteromalidae) to Drosophila simulans (Diptera: Drosophilidae). Heredity 82:163-9.

Min KT, Benzer S. 1997. Wolbachia, normally a symbiont of Drosophila, can be virulent, causing degeneration and early death. Proc Nat Acad Sci USA 94: 10792-6.

Mouton LH, Henri M, Boulétreau F, Vavre E. 2006. Effect of temperature on Wolbachia density and impact on cytoplasmic incompatibility. Parasitology 132:49-56.

O’Neill SL, Giordano R, Colbert AMF, Karr TL, Robertson HM. 1992. 16S rRNA phylogenetic analysis of the bacterial endosymbionts associated with cytoplasmic incompatibility in insects. Proc Nat Acad Sci USA 89: 2699-702.

Poinsot D, Bourtzis K, Markakis G, Savakis, C, Merçot H. 1998. Wolbachia transfer from Drosophila melanogaster into $D$. simulans host effect and cytoplasmic incompatibility relationships. Genetics 150:227-37.

Rousset F, Bouchon D, Pintureau B, Juchault P, Solignac M. 1992. Wolbachia endosymbionts responsible for various alterations of sexuality in arthropods. Proc R Soc London B 250:91-8.

Rousset F, Stordeur E de. 1994. Properties of Drosophila simulans strains experimentally infected by different clones of the bacterium Wolbachia. Heredity 72:325-31.

Rigaud T, Juchault. 1995. Success and failure of horizontal transfer of feminizing Wolbachia endosymbionts in woodlice. J Evol Biol 8:249-55.

Ruang-Areerate T, Kittayapong P. 2006. Wolbachia transinfection in Aedes aegypti: a potential gene driver of dengue vectors. Proc Natl Acad Sci USA 103:12534-9.

Schilthuizen M, Stouthamer R. 1997. Horizontal transmission of parthenogenesis-inducing microbes in Trichogramma wasps. Proc R Soc London B 264:361-6.

Sinkins SP, Braig HR, O’Neill SL. 1995. Wolbachia superinfections and the expression of cytoplasmic incompatibility. Proc R Soc London B 261:325-30.

Stouthamer R, Luck RF, Hamilton WD. 1990. Antibiotics cause parthenogenetic Trichogramma to revert to sex. Proc Nat Acad Sci USA 87:2424-7.

Werren JH. 1997. Wolbachia run amok. Proc Nat Acad Sci USA 94:11154-5.

Werren JH, Windsor D, Guo LR. 1995. Distribution of Wolbachia among neotropical arthropods. Proc R Soc London B 262:197204

Xi Z, Khoo CC, Dobson SL. 2005. Wolbachia establishment and invasion in an Aedes aegypti laboratory population. Science 310:326-8

Xi Z, Khoo CC, Dobson SL. 2006. Interspecific transfer of Wolbachia into the mosquito disease vector Aedes albopictus. Proc Biol Sci 273:1317-22. 